\title{
Half-metallic antiferromagnets in thiospinels
}

\author{
Min Sik Park and B. I. Min \\ Department of Physics, Pohang University of Science and Technology, Pohang 790-784, Korea
}

(October 29, 2018)

\begin{abstract}
We have theoretically designed the half-metallic (HM) antiferromagnets (AFMs) in thiospinel systems, $\mathrm{Mn}(\mathrm{CrV}) \mathrm{S}_{4}$ and $\mathrm{Fe}_{0.5} \mathrm{Cu}_{0.5}\left(\mathrm{~V}_{0.5} \mathrm{Ti}_{1.5}\right) \mathrm{S}_{4}$, based on the electronic structure studies in the local-spin-density approximation (LSDA). We have also explored electronic and magnetic properties of parent spinel compounds of the above systems; $\mathrm{CuV}_{2} \mathrm{~S}_{4}$ and $\mathrm{CuTi}_{2} \mathrm{~S}_{4}$ are found to be $\mathrm{HM}$ ferromagnets in their cubic spinel structures, while $\mathrm{MnCr}_{2} \mathrm{~S}_{4}$ is a ferrimagnetic insulator. We have discussed the feasibility of material synthesis of HM-AFM thiospinel systems.
\end{abstract}

PACS numbers: 71.20.Be, 75.25.+Z, 75.50.Ee

Since the first theoretical report of Heusler halfmetallic (HM) ferromagnet NiMnSb by de Groot et al. [1], much effort has been devoted to developing the HM magnetic materials, in which the conduction electrons at the Fermi level $\mathrm{E}_{\mathrm{F}}$ are $100 \%$ spin-polarized [2]. Especially, the HM antiferromagnet (AFM) attracts great attention because it is a non-magnetic metal but its conduction electrons are $100 \%$ spin-polarized. It can be used as a probe of the spin-polarized scanning tunneling microscope without perturbing the spin-character of samples. Further, the HM-AFM is expected to play a vital role in the future spintronic devices that utilize the spin polarization of the carriers.

The first HM-AFM, $\mathrm{V}_{7} \mathrm{MnFe}_{8} \mathrm{Sb}_{7} \mathrm{In}$, which is a derivative of the Heusler compound, was proposed by van Leuken and de Groot [3]. Anther possibility was suggested by Pickett [1] in the double perovskite system such as $\mathrm{La}_{2} \mathrm{VMnO}_{6}$. In this case, $\mathrm{V}$ and $\mathrm{Mn}$ have antiferromagnetically aligned magnetic moments that exactly cancel each other. To date, there has been no successful experimental realization of the HM-AFM.

The thiospinel $\mathrm{FeCr}_{2} \mathrm{~S}_{4}$ in its metallic phase has the HM ferrimagnetic state with nominal valence configurations of $\mathrm{Fe}^{2+}\left(d^{6}\right)$ and $\mathrm{Cr}^{3+}\left(d^{3}\right)$ [5] 7]. The magnetic moments of Fe and $\mathrm{Cr}$ are $4 \mu_{B}$ and $-3 \mu_{B}$, respectively, which produce the integer total magnetic moment of $-2 \mu_{B}$ per formula unit. From this, one can expect that $\mathrm{FeV}_{2} \mathrm{~S}_{4}$ becomes a HM-AFM, since $\mathrm{V}$ has one less electron than $\mathrm{Cr}$ and so the magnetic moment of two $\mathrm{V}^{3+}\left(d^{2}: \mathrm{S}=1\right)$ ions would cancel that of $\mathrm{Fe}^{2+}\left(d^{6}: \mathrm{S}=2\right)$, still possessing the HM property. Indeed the local-spindensity approximation (LSDA) band calculation yields the HM-AFM electronic structure of $\mathrm{FeV}_{2} \mathrm{~S}_{4}$ [8]. Unfortunately, $\mathrm{FeV}_{2} \mathrm{~S}_{4}$ does not exist in the cubic spinel structure but in the hexagonal NiAs structure (Cr $r_{3} S_{4}$-type) with a complicated magnetic configuration [9]. So the above expectation does not work for $\mathrm{FeV}_{2} \mathrm{~S}_{4}$ in nature.

Motivated by the above expectation, we attempt to search for the HM-AFM in other thiospinel compounds. Most of the thiospinel compounds of $A B_{2} \mathrm{~S}_{4}$-type ( $A, B$ : transition metals) with cubic structure have a ferrimagnetic ground state. Usually, the magnetic moment of the $A$ ion in the tetrahedral site is antiferromagneti- cally polarized with that of the $B$ ion in the octahedral site. Under this circumstance, there are some pairs of $A$ and $B$ which give rise to the exactly cancelled magnetic moment in $A B_{2} \mathrm{~S}_{4}$. Moreover, some spinels have the HM electronic structures, as in $\mathrm{FeCr}_{2} \mathrm{~S}_{4}$. Then, by choosing proper pairs which satisfy these two conditions, one can devise the thiospinels with the HM-AFM nature. One possible pair is $\mathrm{Mn}\left(5 \mu_{\mathrm{B}}\right)$ at A-site and mixed cations $\mathrm{Cr}\left(3 \mu_{\mathrm{B}}\right)-\mathrm{V}\left(2 \mu_{\mathrm{B}}\right)$ at B-site. Another possible pair is $\mathrm{Fe}_{0.5}\left(2.5 \mu_{\mathrm{B}}\right)-\mathrm{Cu}_{0.5}\left(0.0 \mu_{B}\right)$ at A-site and $\mathrm{V}_{0.5}\left(1 \mu_{\mathrm{B}}\right)$ $\mathrm{Ti}_{1.5}\left(1.5 \mu_{\mathrm{B}}\right)$ at B-site. Even though a pair like $\mathrm{Fe}\left(4 \mu_{\mathrm{B}}\right)$ and $\mathrm{V}\left(2 \mu_{\mathrm{B}}\right)$ can be chosen, they are not crystallized in the cubic spinel structure, as discussed above. For the same reason, the pairs, such as $\left[\mathrm{Co}\left(3 \mu_{\mathrm{B}}\right)\right.$ and $\operatorname{Ti}\left(1 \mu_{\mathrm{B}}\right)$ $\left.\mathrm{V}\left(2 \mu_{\mathrm{B}}\right)\right]$, and $\left[\mathrm{Ni}\left(2 \mu_{\mathrm{B}}\right)\right.$ and $\left.\operatorname{Ti}\left(1 \mu_{\mathrm{B}}\right)\right]$, are not suitable for a possible HM-AFM, because $\mathrm{Ti}$ and $\mathrm{V}$ form thiospinels only with $\mathrm{Cu}[10]$.

In this work, we will propose two candidates of HM-AFM in thiospinels with cubic spinel structure: $\mathrm{Mn}[\mathrm{CrV}] \mathrm{S}_{4}$ and $\mathrm{Fe}_{0.5} \mathrm{Cu}_{0.5}\left[\mathrm{~V}_{0.5} \mathrm{Ti}_{1.5}\right] \mathrm{S}_{4}$. We show that electronic structures of these thiospinels have the HMAFM nature, by using the LSDA on the basis of the linearized muffin-tin orbital (LMTO) band method. The von Barth-Hedin form of the exchange-correlation potential is utilized.

$M n[C r V] S_{4}$ - We have performed the LSDA band calculation for a hypothetical spinel $\mathrm{Mn}[\mathrm{CrV}] \mathrm{S}_{4}$. We used two formula units in the primitive unit cell. The primitive unit cell is formed by a cubic closed-packed (fcc) array of $\mathrm{S}$ atoms, in which one eighth of the tetrahedral and onehalf of the octahedral interstitial sites are occupied by cations. S atoms have four-fold coordination, formed by three octahedral cations and one tetrahedral cation. For the LMTO calculation, four empty spheres are considered in the interstitial sites to enhance the packing ratio.

Figure 11 shows that $\mathrm{Mn}[\mathrm{CrV}] \mathrm{S}_{4}$ has the HM-AFM ground state at the lattice constant $a=10.110 \AA$ which is the experimental lattice constant of $\mathrm{MnCr}_{2} \mathrm{~S}_{4}$. Spins of $\mathrm{Cr}$ and $\mathrm{V}$ are ferromagnetically aligned, but they are antiferromagnetically aligned to those of Mn. The energy gap is evident near $E_{F}$ in the spin-up density of states (DOS), and the DOS at $\mathrm{E}_{\mathrm{F}}$ originates mainly from spindown V-3d ( $\sim$ two $t_{2 g}$ electrons) states which will con- 


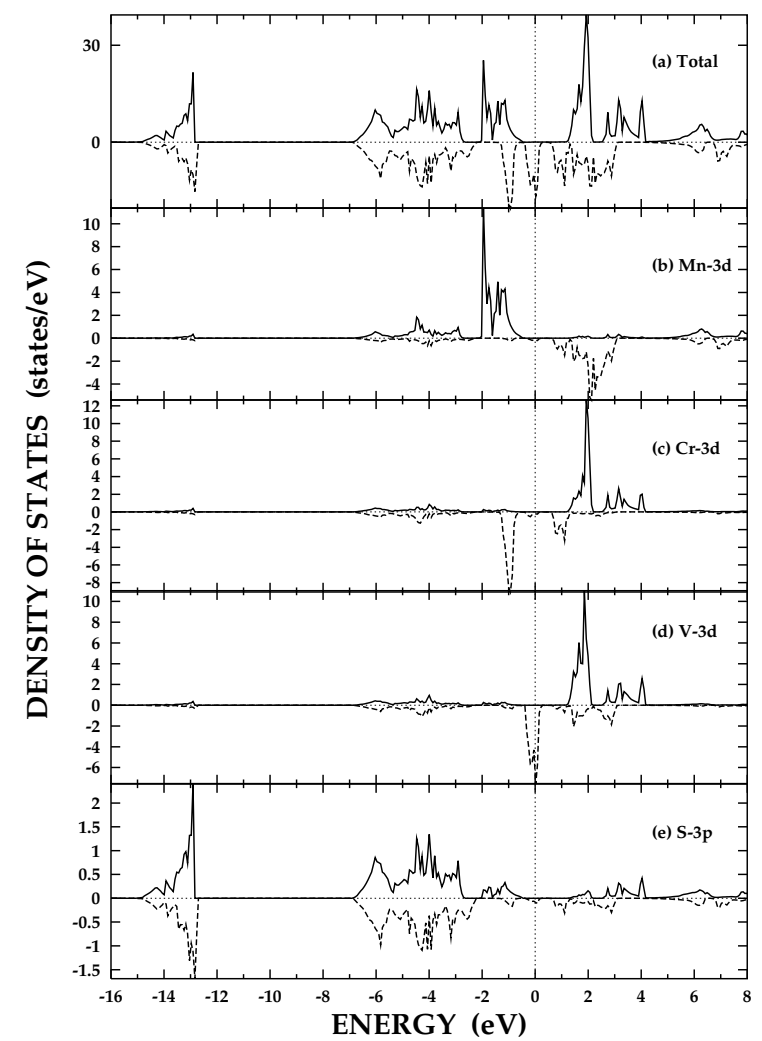

FIG. 1. Total and projected local density of states (PLDOS) of $\mathrm{Mn}[\mathrm{CrV}] \mathrm{S}_{4}$.

tribute to the metallic conductivity. Five spin-up Mn-3d states and three spin-down $\mathrm{Cr}-t_{2 g}$ states are fully occupied. Note that small spin-down states are induced in $\mathrm{Mn}$ and $\mathrm{Cr}$ near $\mathrm{E}_{\mathrm{F}}$ by the hybridization with those of $\mathrm{V}$. The occupied DOS suggests that $\mathrm{Mn}[\mathrm{CrV}] \mathrm{S}_{4}$ has a nominal valence configuration of $\mathrm{Mn}^{2+}[\mathrm{CrV}]^{3+} \mathrm{S}_{4}^{2-}$, which corresponds to the normal spinel structure. By counting the small magnetic moments of S and empty spheres (ES), the total magnetic moment becomes zero in the unit cell which is a nature of the HM-AFM (Table [1).

$\mathrm{MnCr}_{2} \mathrm{~S}_{4}$, the end member of $\mathrm{Mn}[\mathrm{CrV}] \mathrm{S}_{4}$, is known to be a ferrimagnetic insulator with $T_{C}=80 \mathrm{~K}$ [11] and with the total magnetic moment $\sim 1 \mu_{B}$ [12]. Neutron diffraction experiment at $4.2 \mathrm{~K}$ provided that $\mathrm{Cr}$ and $\mathrm{Mn}$ have local magnetic moments of $3 \mu_{B}$ and $4.7 \mu_{B}$, respectively [13]. Our LSDA band calculation yields the insulating ground state (Fig. 2) with local magnetic moments of $3.13 \mu_{B}$ and $4.56 \mu_{B}$ for $\mathrm{Cr}$ and $\mathrm{Mn}$, respectively, which agrees well with experimental results (Table I). The insu-

TABLE I. Total and local spin magnetic moments in $\mu_{B}$ of $\mathrm{Mn}[\mathrm{CrV}] \mathrm{S}_{4}$ (MCVS) and $\mathrm{MnCr}_{2} \mathrm{~S}_{4}$ (MCS). ES denotes the empty sphere.

\begin{tabular}{ccccccc}
\hline \hline & Total & $\mathrm{Mn}$ & $\mathrm{Cr}$ & $\mathrm{V}$ & $\mathrm{S}$ & $\mathrm{ES}$ \\
\hline MCVS & 0.00 & -4.55 & 3.17 & 1.99 & -0.11 & -0.09 \\
MCS & 1.00 & -4.56 & 3.13 & & -0.13 & -0.09 \\
\hline \hline
\end{tabular}

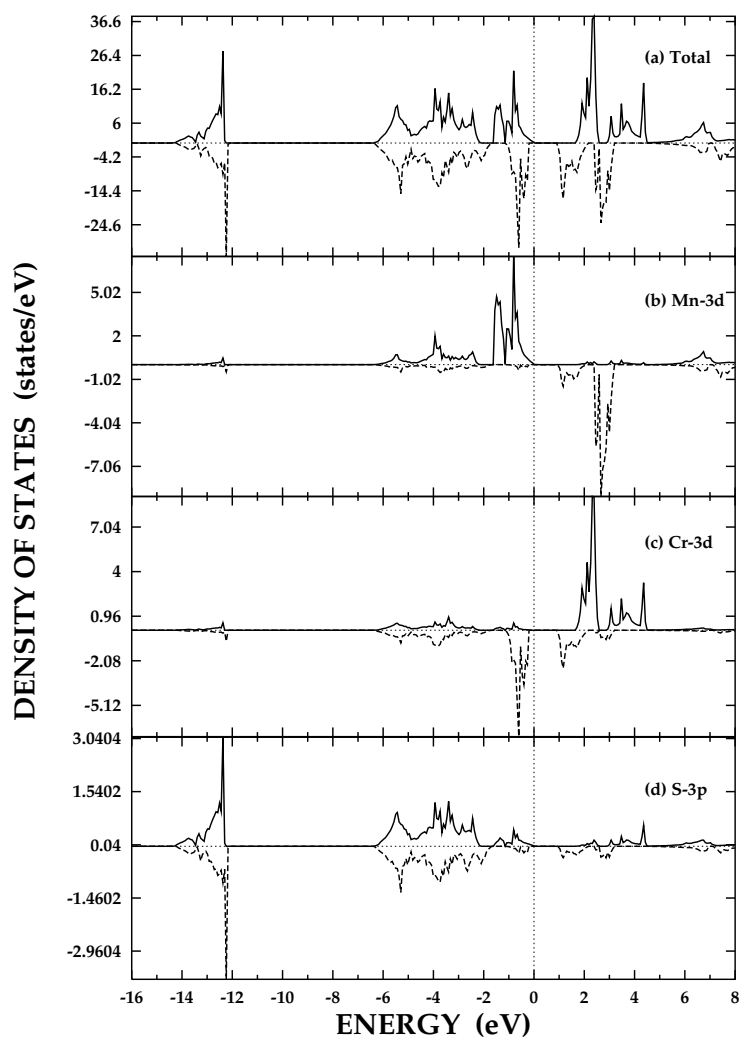

FIG. 2. Total and PLDOS of $\mathrm{MnCr}_{2} \mathrm{~S}_{4}$.

lating property comes from the Mn $\left(e_{g}^{2} t_{2 g}^{3}\right)$ exchange split spin polarized energy gap and the $\mathrm{Cr}\left(t_{2 g}^{3}\right)$ crystal field split energy gap. The ferrimagnetism in $\mathrm{MnCr}_{2} \mathrm{~S}_{4}$ can be understood in terms of the ordinary kinetic superexchange interaction. It is thus evident that the HM-AFM $\mathrm{Mn}[\mathrm{CrV}] \mathrm{S}_{4}$ results from the insertion of $\mathrm{V}-3 d$ band in the gap region of $\mathrm{MnCr}_{2} \mathrm{~S}_{4}$ with the magnetic moment parallel to that of $\mathrm{Cr}$.

Unfortunately, the other end member $\mathrm{MnV}_{2} \mathrm{~S}_{4}$ exists in the $\mathrm{Cr}_{3} S_{4}$-type structure [14]. It has been reported that the spinel phase of $\mathrm{Mn}\left[\mathrm{Cr}_{2-\mathrm{x}} \mathrm{V}_{\mathrm{x}}\right] \mathrm{S}_{4}$ is formed successfully up to $x=0.6$ with decreasing magnetic moment from $\sim 1 \mu_{B}$ at $x=0.0$ to $\sim 0.4 \mu_{B}$ at $x=0.6$ [15]. From this, one can extrapolate zero magnetic moment at $x=1.0$ in accordance with the present LSDA band result. Only when the spinel structure with substituting $\mathrm{V}$ for $\mathrm{Cr}$ in $\mathrm{MnCr}_{2} \mathrm{~S}_{4}$ is retained, the HM-AFM Mn[CrV] $\mathrm{S}_{4}$ could be synthesized. The above experimental result may indicate that the bulk spinel phase of $\mathrm{Mn}\left[\mathrm{Cr}_{2-\mathrm{x}} \mathrm{V}_{\mathrm{x}}\right] \mathrm{S}_{4}$ would be unstable over $x=0.6$. Then the possible attempt would be an artificial fabrication of $\mathrm{Mn}[\mathrm{CrV}] \mathrm{S}_{4}$ film on appropriate substrates, which is desirable to be tested experimentally.

$F e_{0.5} C u_{0.5}\left[V_{0.5} T i_{1.5}\right] S_{4}$ - We have mentioned that $\mathrm{FeV}_{2} \mathrm{~S}_{4}$, if it exists in the cubic spinel form, would be a strong candidate for the HM-AFM. We have also seen that $\mathrm{V}$ and $\mathrm{Ti}$ form thiospinels only with $\mathrm{Cu}: \mathrm{CuV}_{2} \mathrm{~S}_{4}$ and $\mathrm{CuTi}_{2} \mathrm{~S}_{4}$ [10]. On the basis of this, we have explored the combination of $\mathrm{Fe}, \mathrm{Cu}, \mathrm{V}$, and $\mathrm{Ti}$ to make a 


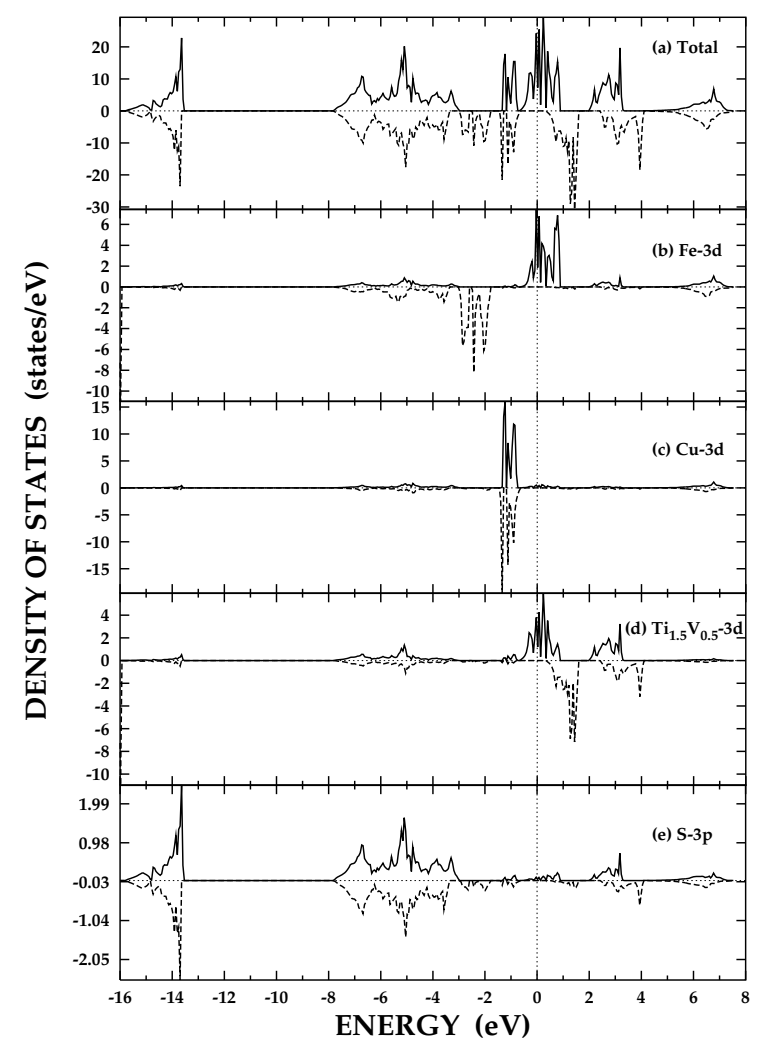

FIG. 3. Total and PLDOS of $\mathrm{Fe}_{0.5} \mathrm{Cu}_{0.5}\left[\mathrm{~V}_{0.5} \mathrm{Ti}_{1.5}\right] \mathrm{S}_{4}$.

thiospinel possessing both the HM and the AFM nature: $\mathrm{Fe}_{0.5} \mathrm{Cu}_{0.5}\left[\mathrm{~V}_{0.5} \mathrm{Ti}_{1.5}\right] \mathrm{S}_{4}$ (FCVTS). We have performed the LSDA electronic structure calculation for FCVTS by replacing $\mathrm{V}_{0.5} \mathrm{Ti}_{1.5}$ with a virtual atom having atomic number 22.25. The lattice constant of $a=9.8865 \AA$ is used which is interpolated from end members, $\mathrm{CuV}_{2} \mathrm{~S}_{4}$ and $\mathrm{CuTi}_{2} \mathrm{~S}_{4}$. As shown in Fig. 3, FCVTS really has the HM-AFM electronic structure. Ten $\mathrm{Cu} 3 d$-states and five spin-down Fe $3 d$-states are fully occupied. The DOS at $\mathrm{E}_{\mathrm{F}}$ originates from spin-up Fe $3 d\left(\sim e_{g}^{1}\right)$ and $\mathrm{V}_{0.5} \mathrm{Ti}_{1.5}$ $3 d\left(\sim t_{2 q}^{2}\right)$ states. The calculated magnetic moments in Table. III reflect that the valence configuration of FCVTS corresponds to $\mathrm{Fe}_{0.5}^{2+} \mathrm{Cu}_{0.5}^{1+}\left[\mathrm{V}_{0.5} \mathrm{Ti}_{1.5}\right]^{3.25+} \mathrm{S}_{4}^{2-}$ with zero total magnetic moment. The magnetic mechanism in FCVTS can be understood in a similar way to the case in $\mathrm{Fe}_{0.5} \mathrm{Cu}_{0.5} \mathrm{Cr}_{2} \mathrm{~S}_{4}$ [5]

Now, let us discuss the possibility of synthesizing FCVTS. We first consider parent thiospinels, $\mathrm{CuV}_{2} \mathrm{~S}_{4}$ and $\mathrm{CuTi}_{2} \mathrm{~S}_{4}$, both of which have metallic nature 16 .

TABLE II. Total and local spin magnetic moments in $\mu_{B}$ of $\mathrm{Fe}_{0.5} \mathrm{Cu}_{0.5}\left[\mathrm{~V}_{0.5} \mathrm{Ti}_{1.5}\right] \mathrm{S}_{4}$ (FCVTS), cubic spinel $\mathrm{CuV}_{2} \mathrm{~S}_{4}$ (CVS), and $\mathrm{CuTi}_{2} \mathrm{~S}_{4}$ (CTS).

\begin{tabular}{ccccccccc}
\hline \hline & Total & $\mathrm{Fe}$ & $\mathrm{Cu}$ & $\mathrm{V}_{0.5} \mathrm{Ti}_{1.5}$ & $\mathrm{~V}$ & $\mathrm{Ti}$ & $\mathrm{S}$ & $\mathrm{ES}$ \\
\hline FCVTS & 0.00 & -3.57 & -0.13 & 1.08 & & & -0.07 & 0.03 \\
CVS & 3.00 & & -0.28 & & 1.89 & & 0.12 & 0.00 \\
CTS & 1.00 & & -0.17 & & & 0.66 & 0.04 & 0.00 \\
\hline \hline
\end{tabular}

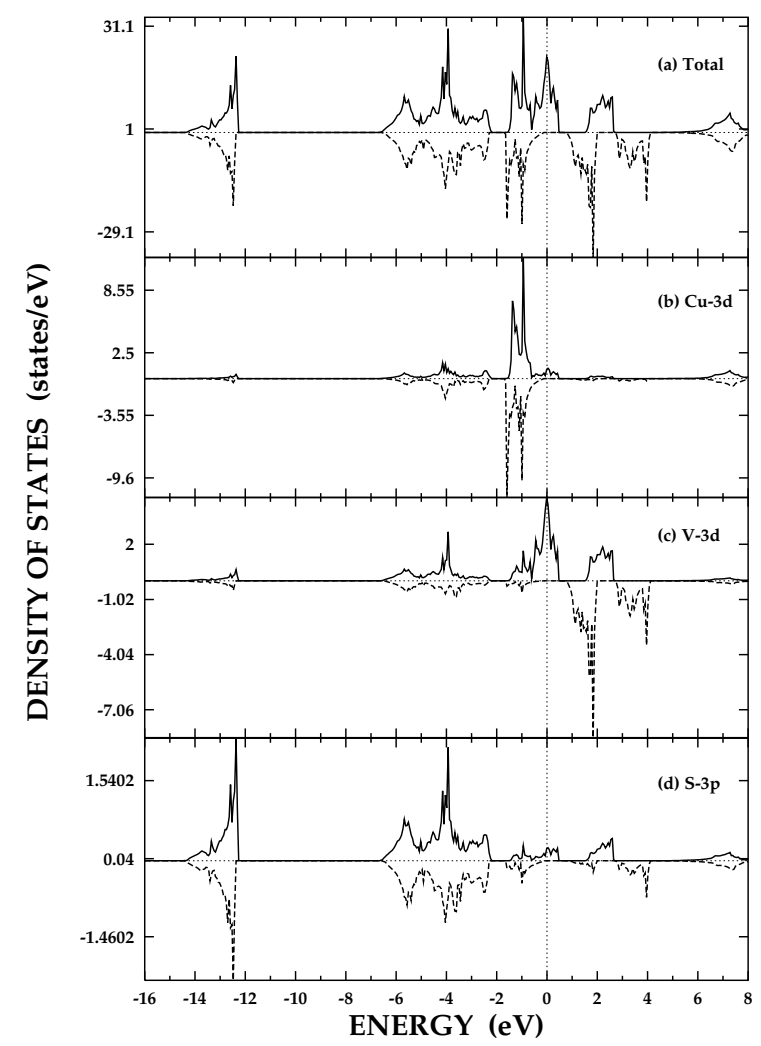

FIG. 4. Total and PLDOS of $\mathrm{CuV}_{2} \mathrm{~S}_{4}$.

The LSDA band calculation shows that the $\mathrm{CuV}_{2} \mathrm{~S}_{4}$ has a HM ground state in its cubic spinel structure (Fig. (4). But, this result does not coincide with experimental results for $\mathrm{CuV}_{2} \mathrm{~S}_{4}$ having no spontaneous magnetic property. In fact, $\mathrm{CuV}_{2} \mathrm{~S}_{4}$ has a $\mathrm{CDW}$-type phase transition below $90 \mathrm{~K}$. Accordingly, the cubic unit cell is transformed to a tetragonal structure which is induced by the Jahn-Teller local small distortion at the $\mathrm{V}$ site [17 21]. Our result suggests that if $\mathrm{CuV}_{2} \mathrm{~S}_{4}$ exists in the cubic spinel structure, it will have a HM ferromagnetic ground state. The oxidation state of $\mathrm{Cu}$ atoms of $\mathrm{CuV}_{2} \mathrm{~S}_{4}$ is closer to $\mathrm{Cu}^{+}$222]. The bands near $\mathrm{E}_{\mathrm{F}}$ are predominantly of $\mathrm{V} 3 d$ character, so that the metallic nature of $\mathrm{CuV}_{2} \mathrm{~S}_{4}$ comes mainly from the $\mathrm{V}$ atom 222.

The CDW-type phase transition can be suppressed by the random occupation of the octahedral sites in the spinel structure. Indeed, $\mathrm{Cu}\left[\mathrm{V}_{1.9} \mathrm{Ti}_{0.1}\right] \mathrm{S}_{4}$ showed no evidence of a phase transition due to random occupation of the octahedral sites by the V-Ti mixture 23. $\mathrm{CuV}_{2} \mathrm{~S}_{4}$ has a large susceptibility due to considerable exchange enhancement [23]. The ferromagnetism in the Cr-mixed $\mathrm{CuV}_{2} \mathrm{~S}_{4}$ compound $\mathrm{CuV}_{2-\mathrm{x}} \mathrm{Cr}_{\mathrm{x}} \mathrm{S}_{4}$ at low $\mathrm{Cr}$ concentrations is considered to come from the large susceptibility of $\mathrm{CuV}_{2} \mathrm{~S}_{4}$ 23]. Therefore, one can also expect that, if some of $\mathrm{Cu}$ atoms are replaced by $\mathrm{Fe}$ atoms, $(\mathrm{FeCu}) \mathrm{V}_{2} \mathrm{~S}_{4}$ would become a ferromagnet.

$\mathrm{CuTi}_{2} \mathrm{~S}_{4}$ shows a metallic behavior 24] and the weak magnetism 25. Our LSDA band calculation also indicates that both the metallic nature and the weak mag- 


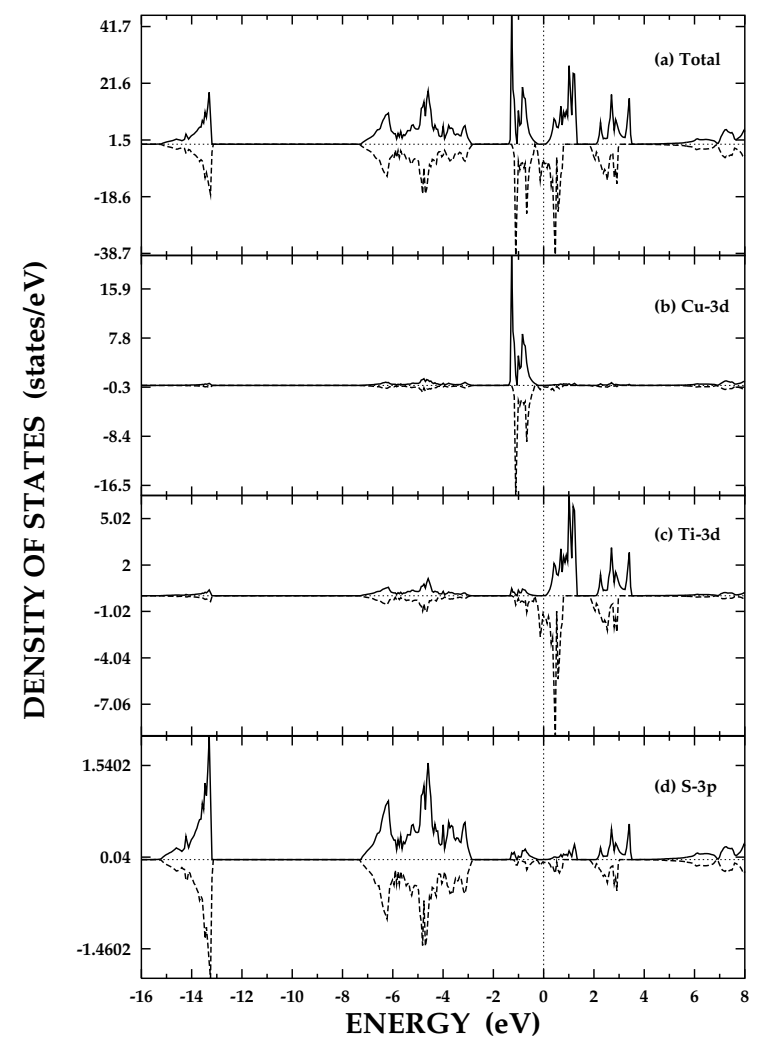

FIG. 5. Total and PLDOS of $\mathrm{CuTi}_{2} \mathrm{~S}_{4}$.

netism of $\mathrm{CuTi}_{2} \mathrm{~S}_{4}$ result from $3 d$-bands of Ti ions (Table. II). As shown in Fig. 司, the $\mathrm{CuTi}_{2} \mathrm{~S}_{4}$ also has a HM ferromagnetic ground state. The electronic configuration of $\mathrm{Cu}$ at the A-site is considered to be close to $e_{g}^{4} t_{2 g}^{6}$ $(\mathrm{S}=0)$. Combining the above features, one can make a metallic $\mathrm{Cu}\left[\mathrm{V}_{0.5} \mathrm{Ti}_{1.5}\right] \mathrm{S}_{4}$ having a large susceptibility. Then by mixing Fe ions with $\mathrm{Cu}\left[\mathrm{V}_{0.5} \mathrm{Ti}_{1.5}\right] \mathrm{S}_{4}$ to have exact cancellation of magnetic moments, one can possibly make HM-AFM FCVTS.

In conclusion, we have predicted that $\mathrm{Mn}[\mathrm{CrV}] \mathrm{S}_{4}$ and FCVTS are candidates for the HM-AFM in thiospinels, based on the LMTO band results in the LSDA. $\mathrm{Mn}[\mathrm{CrV}] \mathrm{S}_{4}$ and FCVTS satisfy two criteria for the HMAFM: the antiferromagnetic couplings between magnetic moments and the HM property. The possibility of synthesizing HM-AFMs is discussed by considering electronic, transport, and magnetic properties of parent thiospinel compounds. $\mathrm{MnCr}_{2} \mathrm{~S}_{4}$ found to be a ferrimagnetic insulator in agreement with experiment, while $\mathrm{CuV}_{2} \mathrm{~S}_{4}$ and $\mathrm{CuTi}_{2} \mathrm{~S}_{4}$ are found to be HM ferromagnets in their cubic spinel structures.

Acknowledgments - This work was supported by the KOSEF through the eSSC at POSTECH and in part by the BK21 Project.
[1] R. A. de Groot and F. M. Mueller, P. G. van Engen, and K. H. J. Buschow, Phys. Rev. Lett. 50, 2024 (1983).

[2] see the review by V. Yu. Irkhin and M. I. Katsnel'son, Usp. Fiz. Nauk 164, 705 (1994) [Sov. Phys. Usp. 37, 659 (1994)].

[3] H. van Leuken and R. A. de Groot, Phys. Rev. Lett, 74, 1171 (1995).

[4] W. E. Pickett, Phys. Rev. B 57, 10613 (1998).

[5] M. S. Park, S. K. Kwon, S. J. Youn, and B. I. Min, Phys. Rev. B 59, 10018 (1999). The ground state of $\mathrm{FeCr}_{2} \mathrm{~S}_{4}$ is an insulator due to the Jahn-Teller distortion below 10 $\mathrm{K}$.

[6] J. -S. Kang, S. J. Kim, C. S. Kim, C. G. Olson, and B. I. Min, Phys. Rev. B 63, 144412 (2001).

[7] E. Z. Kurmaev, A. V. Postnikov, H. M. Palmer, C. Greaves, S. Bartkowski, V. Tsurkan, M. Demeter, D. Hartmann, M. Neumann, D. A. Zatsepin, V. R. Galakhov, S. N. Shamin, V. Trofimova, J. Phys.: Condens. Matter 12, 5411 (2000).

[8] M. S. Park and B. I. Min, unpublished.

[9] A. V. Powell, C. Ritter and P. Vaqueiro, J. Solid State Chem. 144, 372 (1999).

[10] A. Wold and K. Dwight, Solid State Chemistry: Synthesis, Structure and Properties of Selected Oxides and Sulfides, Chapman and Hall, New York, 1993, ch11.

[11] F. K. Lotgering, J. Phys. Chem. Solids. 29, 699 (1968).

[12] V.Tsurkan, M. Demeter, B. Schneider, D. Hartmann, M. Neumann, Solid State Commun. 114, 149 (2000).

[13] N. Menyuk, K. Dwight, A. Wold, J. Appl. Phys. 36, 1088 (1965).

[14] M. Nogues, M. Mejai and L. Goldstein, J.Phys. Chem. Solids, 40, 375 (1979).

[15] L. Goldstein, P. Gibart, M. Mejai and M. Perrin, Physica 86-88B, 893 (1977).

[16] R. J. Bouchard, P. A. Russo and A. Wold, Inorg. Chem. 4, 685 (1965).

[17] Y.Yoshikawa, S. Wada, K. Miyatani, T. Tanaka and M. Miyamoto, Phys. Rev. B 55, 74 (1997).

[18] R. M. Fleming, F. J. Disalvo, R. J. Cava and J. V. Waszczak, Phys. Rev. B 24, 2850 (1981).

[19] J. Mahy, D. Colaitis, D. Van Dyck and S. Amelinckx, J. Solid State Chem. 68, 320 (1987).

[20] T. Hagino, Y. Seki, S. Takayanagi, N. Wada and S. Nagata, Phys. Rev. B 49, 6822 (1994).

[21] T. Ohno, Y. Kishimoto, Physica B 259-261, 971 (1999).

[22] Z. W. Lu, B. M. Klein, E. Z. Kurmaev, V. M. Cherkashenko, V. R. Galakhov, S. N. Shamin, Yu. M. Yarmoshenko, V. A. Trofimova, St. Uhlenbrock, M. Neumann, T. Furubayashi, T. Hagino and S. Nagata, Phys. Rev. B 53, 9626 (1996).

[23] F. J. DiSalvo and J. V. Waszczak, Phys. Rev. B 26, 2501 (1982).

[24] N. Matsumoto, T. hagino, K. Taniguchi, S. Chikazawa, S. Nagata, Physica B 284-288, 1978 (2000).

[25] T. Koyama, H. Sugita, S. Wada, K. Miyatani, H. Tanaka, M. Ishikawa, Physica B 284-288, 1513 (2000). 